Case Report

\title{
ORAL PYOGENIC GRANULOMA: A CASE REPORT
}

\author{
Swati Kejriwal ${ }^{1}$, Rahul Bhandary ${ }^{2} \&$ Biju Thomas $^{3}$ \\ ${ }^{1}$ P.G. Student, ${ }^{2}$ Professor, ${ }^{3}$ Professor \& HOD, Department of Periodontics \\ A.B. Shetty Memorial Institute of Dental Sciences, Nitte University, Deralakatte, Mangalore - 575018 \\ Correspondence: \\ Swati Kejriwal \\ P.G. Student, Department of Periodontics, A.B.Shetty M emorial Institute of Dental Sciences \\ Nitte University, Deralakatte, Mangalore - 575 018. Karnataka. E-mail : drswatikejriwal@gmail.com
}

\begin{abstract}
:
Pyogenic granuloma is an inflammatory hyperplasia affecting the oral tissues. It is a tumour-like growth of the oral cavity, which usually arises in response to nonspecific infection. Because of the high frequency of pyogenic granuloma in the oral cavity, this case report describes a pyogenic granuloma in a 59-year-old male patient, discussing the clinical features and histopathologic features that distinguish this lesion from other similar oral mucosa lesions and also the successful management of the lesion.
\end{abstract}

Keywords: Pyogenic granuloma, Hyperplasia, granuloma pyogenicum.

\section{Introduction:}

The term "Pyogenic granuloma" or "granuloma pyogenicum" was introduced by Hartzell in 1904. ${ }^{1}$ It is a hyperactive benign inflammatory lesion commonly seen in the oral cavity with gingiva being the most common affected site followed by buccal mucosa, tongue and lips.

It usually arises in response to various stimuli such as lowgrade local irritation, traumatic injury, hormonal factors, or certain kinds of drugs. ${ }^{2}$ Pyogenic granuloma (PG) may occur in all age groups, though it is predominantly seen in young females in the second decade of life because of the hormonal changes in this period. ${ }^{2}$ This article reports the case of a 59-year-old male patient with pyogenic granuloma successfully managed by surgical intervention.

\section{Case Report:}

A 59-year-old male patient reported to the Department of Periodontics with a complaint of growth in the lower right back region of the mouth since 2 months. The growth was initially small in size and slowly growing attaining the

\begin{tabular}{|c|}
\hline Access this article online \\
\hline Quick Response Code \\
\hline \\
\hline
\end{tabular}
present size. The patient gave history of a similar lesion 5 months back for which the patient had undergone surgical excision 3 months back. Patient reported discomfort associated with the growth which increased on chewing food. Medical history was non contributory. Intraoral examination revealed a solitary red exophytic, pedunculated growth on the gingiva measuring about $1.5 \mathrm{x}$ $2 \times 1 \mathrm{~cm}$ present in relation to distal aspect of 47 (Fig. 1). On palpation, inspectory findings were confirmed. The growth was firm on palpation, non tender with absence of discharge. Bleeding on provocation was positive. Oral hygiene was poor with calculus and stains. Complete hemogram showed all blood counts within normal limits. Intraoral periapical radiograph (IOPAR) revealed no abnormalities (Fig. 2). Oral prophylaxis was performed. In addition, chemical plaque control measures were advised in the form of $0.2 \%$ chlorhexidine gluconate mouthwash, to be used twice daily. Excisional biopsy was performed under local anesthesia and sent for histopathologic examination. Histopathological examination showed a hyperplastic, parakeratinized stratified squamous epithelium. The connective tissue was loose fibrillar and comprised of numerous proliferating capillaries, dense mixed inflammatory infiltrate, and extravasated red blood cells (Fig. 3,4). The histopathological examination confirmed the clinical diagnosis of Pyogenic granuloma. The patient was recalled after a week and the excised area was evaluated for healing. Healing was satisfactory. The patient was recalled periodically at two weeks, 1 month, three months and six months. At each visit, oral prophylaxis and oral 
hygiene instructions were reinforced. Even after six months, there was no recurrence of the lesion (Fig.5).

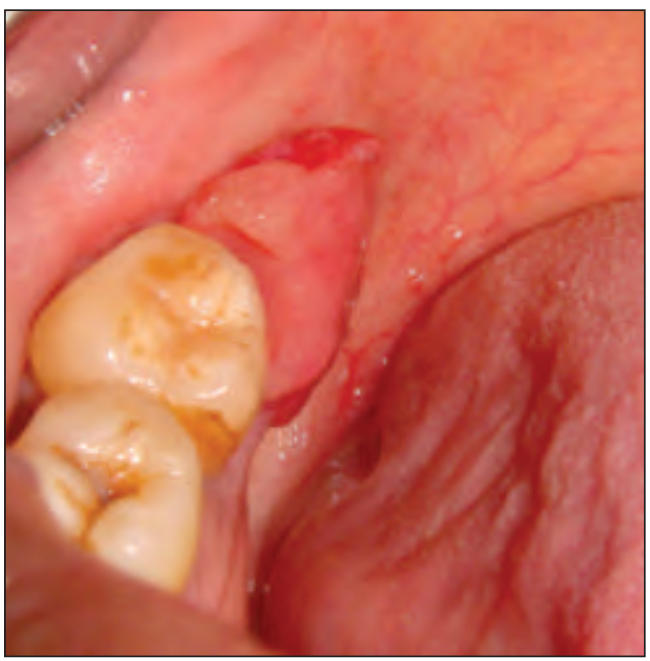

Fig.1: Pre-operative photograph showing the lesion distal to 47

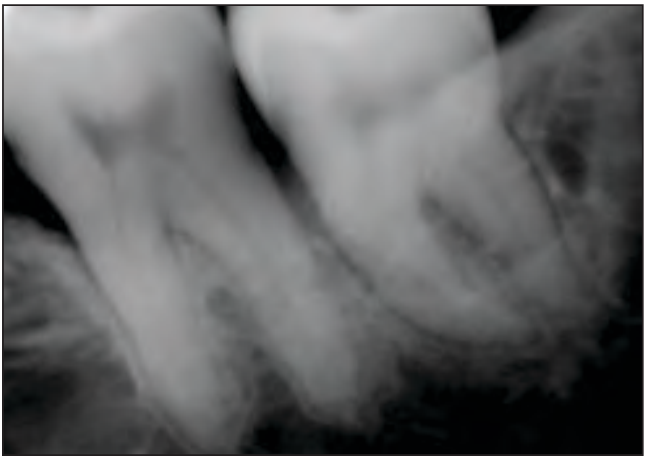

Fig.2: Intraoral periapical radiograph.

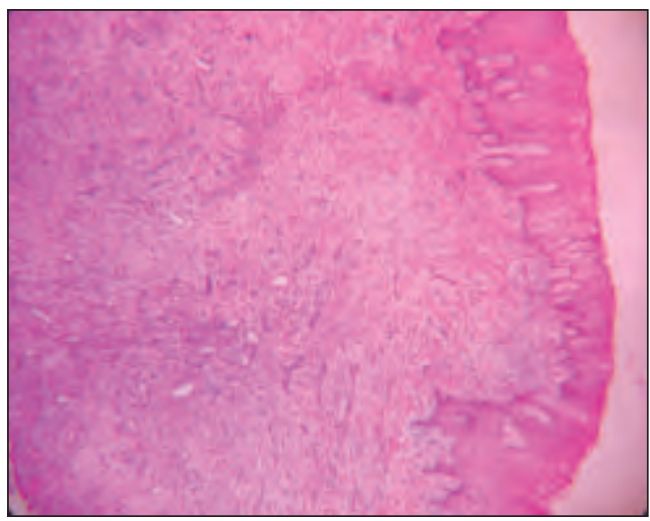

Fig.3: Photomicrograph showing hyperplastic, parakeratinized stratified squamous epithelium and connective tissue with numerous proliferating capillaries, dense mixed inflammatory infiltrate, and extravasated red blood cells ( $H \&$ Estain, 4X magnification).

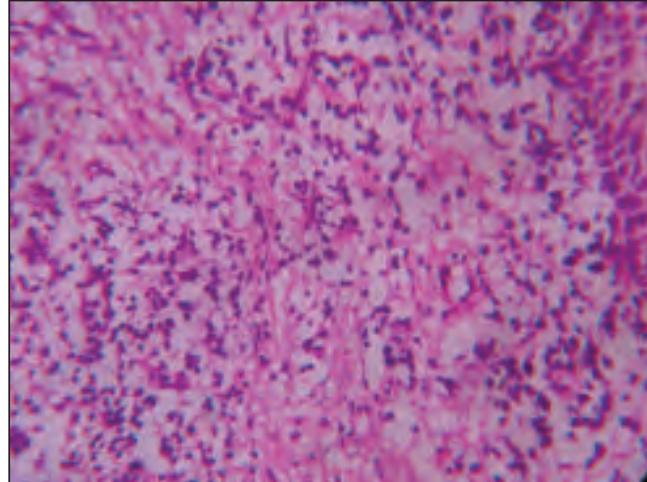

Fig.4: Photomicrograph showing epithelium and connective tissue (H \& Estain, 40X magnification).

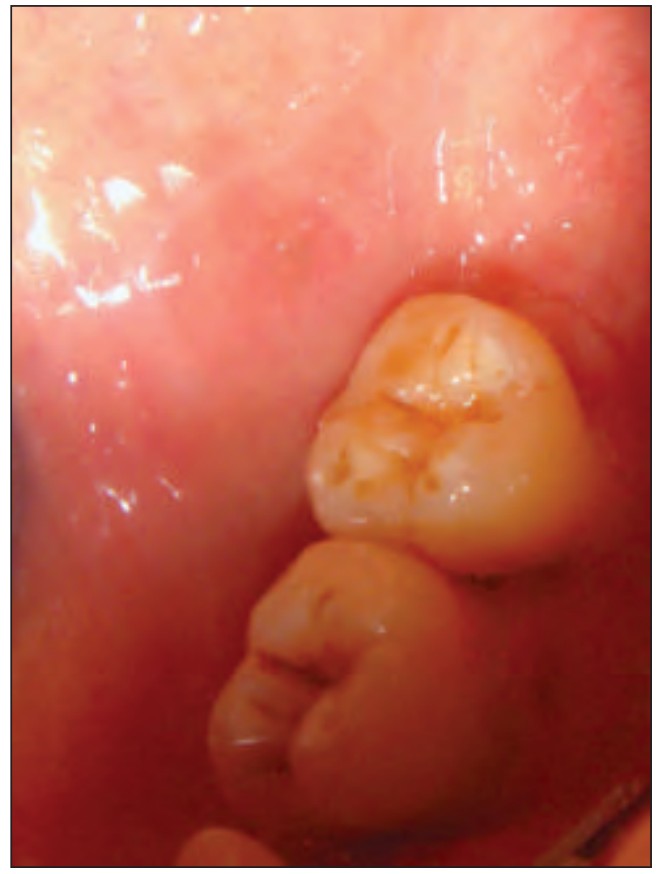

Fig.5: Six months postoperative view

\section{Discussion :}

Gingival enlargement is defined as an increase in size of the gingiva. Depending on etiologic factors, gingival enlargement can be of many types like inflammation, drug induced effects, neoplasm, hormonal imbalance, non specific conditioned enlargement and systemic involvement like Leukemia, Wegener's granulomatosis etc. ${ }^{2}$ Nonspecific conditioned enlargement, or pyogenic granuloma, is a benign, localized mass of exuberant granulation tissue. It is considered as an exaggerated conditioned response to minor trauma or chronic irritation. ${ }^{3}$ However, the term is a misnomer since the condition is not associated with pus formation and does 
not represent a granuloma histologically. ${ }^{4} P G$ is predominantly seen in the second decade of life though it may occur in all age groups. ${ }^{2}$ In contrast, Epivatianos et al reported that the average patient age was 52 years with a peak incidence of occurrence in the sixth decade of life. ${ }^{5}$ In the present case, the patient was 59 years old. Oral PG shows a predilection for the gingiva accounting for $75 \%$ of all casesfollowed by buccal mucosa, tongue and lips. ${ }^{6}$ Here, the lesion was present on the gingiva distal of 47 . Rarely, PG may cause significant bone loss. ${ }^{7}$ Here, IOPAR revealed no abnormalities. Studies have shown that PG may occur as a result of an exaggerated localized connective tissue reaction to a minor injury or any underlying irritation. The irritating factor can be poor oral hygiene, nonspecific infection, over hanging restorations, cheek biting etc. Because of this irritation, the underlying fibrovascular connective tissue becomes hyperplastic and there is proliferation of granulation tissue which leads to the formation of a pyogenic granuloma. ${ }^{8}$ In the present case, patient's oral hygiene was poor. Chronic irritation resulting from accumulated plaque and calculus could have contributed to the development of PG.

\section{References:}

1. Hartzell M B. Granuloma pyogenicum. J Cutan Dis Syph 1904;22:520525.

2. Sumanth Shivaswamy, Nazia Siddiqui, A. Sanjay Jain, Ajit Koshy, Sonal Tambwekar, Akhil Shankar. A rare case of generalized pyogenic granuloma: A case report. Quintessence Int 2011;42:493-499.

3. Ningappa Chinnannavar Sangamesh, Bellguppa Poornima, Kodige Chandrashekar Vidya, Santosh Bhopal Sakri. Extragingival pyogenic granuloma: A rare case report. Journal of the Scientific Society 2013;40(1):49-51.

4. Reet Kamal, Parveen Dahiya, Abhiney Puri. Oral pyogenic granuloma: various concepts of etiopathogenesis. Journal of Oral and Maxillofacial Pathology 2012;16(1):79-82.

5. Epivatianos A, Antoniades D, Zaraboukas T, Zairi E, Poulopoulos A, Kiziridou A, Iordanidis S. Pyogenic granuloma of the oral cavity: comparative study of its clinicopathological and immunohistochemical features. Pathol Int 2005;55:391-7.

6. Hamid Jafarzadeh, Majid Sanatkhani, Nooshin Mohtasham. Oral pyogenic granuloma: a review. Journal of Oral Science 2006;48(4):167175.

7. Goodman-Topper ED, Bimstein E. Pyogenic granuloma as a cause of bone loss in a twelve-year-old child: report of case. ASDC J Dent Child 1994;61:65-67.

8. Pushpendra Kumar Verma, Ruchi Srivastava, HC Baranwal, TP Chaturvedi, Anju Gautam, Amit Singh. Pyogenic Granuloma Hyperplastic Lesion of the Gingiva: Case Reports. Open Dent J. 2012; 6: 153-156.
PG may be confused with other benign and malignant conditions because of its appearance and evolution of growth; hence, biopsy findings are important in establishing diagnosis. ${ }^{2}$ The clinical and histopathological finding led to a final diagnosis of PG. Treatment of PG involves a complete surgical excision. After excision, recurrence rate has been reported in up to $16 \%$ of lesions. Recurrence is believed to result from incomplete excision, failure to remove etiologic factors, or re-injury of the area. Also gingival lesions show a much higher recurrence rate than lesions from other oral mucosal sites. ${ }^{6}$ Here the patient gave history of similar lesion which was excised 3 months back. The patient was recalled for regular maintenance visits, and after 6 months of the treatment there was no recurrence seen.

\section{Conclusion:}

From the present case report it is concluded that pyogenic granuloma can be adequately treated with the correct diagnosis and proper treatment planning. A careful management of the lesion also helps in preventing the recurrence of this benign lesion. 